\title{
PENSAMIENTO CRÍTICO LATINOAMERICANO: LA IMPUGNACIÓN DEL EUROCENTRISMO
}

\author{
EDGARDO LANDER
}

Las diferentes vertientes principales del pensamiento hegemónico sobre y desde América Latina pueden ser caracterizadas como colonial/eurocéntricas. Existe una continuidad básica desde las crónicas de indias, el pensamiento liberal de la independencia, el positivismo y el pensamiento conservador del siglo XIX, la sociología de la modernización, marxismo(s) y desarrollismo en sus diversas versiones durante el siglo XX, y en la actualidad, el neoliberalismo y las disciplinas académicas institucionalizadas en las universidades del continente. Más allá de la diversidad de sus orientaciones y de sus variados contextos históricos es posible identificar en estas corrientes hegemónicas un sustrato colonial que se expresa en la lectura de estas sociedades a partir de la cosmovisión europea y su propósito de transformarlas a imagen y semejanza de las sociedades del Norte que en sucesivos momentos históricos han servido de modelo a imitar. Estas vertientes dominantes del pensamiento latinoamericano han sido la expresión del imaginario de la minoría, élites blancas, generalmente urbanas, que han tenido - y continua teniendo- una mayor identificación y convergencia de intereses con los grupos dominantes en los centros metropolitanos que con las condiciones de vida y aspiraciones de la mayoría de la población.

Sin embargo, se han producido igualmente otras vertientes de pensamiento y otras opciones de conocimiento sobre la realidad del continente que se han hecho en los márgenes, en la defensa de formas ancestrales alternativas del conocer, en la resistencia cultural, o asociadas a luchas políticas y/o procesos movilización popular. Para estas perspectivas el saber, el conocimiento, el método, la construcción del imaginario sobre lo que se ha sido, se es y se puede llegar a ser como pueblos, lejos de ser un exquisito asunto propio de especialistas en epistemología, es una cuestión de medular importancia política y cultural en la medida en que las formas hegemónicas del conocimiento sobre estas sociedades han operado como eficaces artefactos de legitimación y naturalización de la jerarquización y exclusión social que ha prevalecido históricamente en estas cociedades. La descolonización del imaginario y la desuniversalización de las formas coloniales del saber aparecen así como condiciones de toda transformación democrática radical de estas sociedades. Estos han sido asuntos presentes en el debate por lo menos desde las contribuciones de Martí y Mariátegui en las últimas décadas del siglo XIX y primeras del siglo XX. Como lo señaló lúcidamente Darcy Ribeiro en la década de los 60 del siglo pasado:

"De la misma forma en que Europa llevó una variedad de técnicas e invenciones a los pueblos incluidos en su red de dominación... también los introdujo a un instrumental de conceptos, preceptos e idiosincrasia que se refería al mismo tiempo a Europa misma y a los pueblos coloniales. 
Los pueblos coloniales. privados de sus riquezas y del fruto de su trabajo bajo los regímenes coloniales. sufrieron aún más la degradación de asumir como su propia imagen la imagen que no era más que una reflexión de la visión europea del mundo que consideraba a los pueblos colonizados racialmente inferiores porque eran negros. indios, o mestizos... Aún los estratos sociales más brillantes de pueblos no-europeos se acostumbraron a verse a sí mismos y a sus comunidades como una infra-humanidad cuyo destino era el ocupar una posición subalterna debido al simple hecho de que su población era inferior a la europea."

Ha sido fecunda, así como poco asumida en los medios académicos latinoamericanos actuales, la exploración no sólo de interpretaciones de estas sociedades que van a contracorriente de las concepciones dominantes sobre el continente, sino igualmente de otras modalidades del conocer y otras nociones del sujeto y de la relación objeto/sujeto en el proceso del conocimiento. Tres ejemplos son suficientes para ilustrar esta rica herencia. El pensamiento político-académico de las décadas de los 60 y los 70 -época de los debates sobre la dependencia- si bien no logró desprenderse del imaginario (colonial) del desarrollo, significó no sólo rupturas importantes en el rechazo a las particiones dogmáticas que estableció la tradición liberal entre los diferentes ámbitos de lo histórico-social (lo político. lo social, lo económico. lo cultural), sino que igualmente transgredió expresamente las exigencias de objetividad de una ciencia social que pretendía ser valorativamente neutra, al asumir la producción de conocimiento de lo social como una toma de partido, como parte de un compromiso político de transformación social. Desde el punto de vista metodológico la investigación-acción, o imvestigación militante, que tiene como máxima expresión la obra de Orlando Fals Borda y sus colaboradores, rechazando las pretensiones objetivistas del método científico, se propone la investigación como instrumento de transformación, y la práctica de la transformación social como vía privilegiada del conocimiento de lo histórico-social. La pedagogía del oprimido de Paulo Freire expresa esa misma ruptura en el campo pedagógico. Rechazando -por conservadora y reproductora de las relaciones sociales jerárquicas existentesel que el maestro sea poseedor de un conocimiento que deba llevar (con el cual deba "llenar") al educando, Freire propone metodologías de aprendizaje activo, a partir de las propias prácticas y vivencias. El proceso del conocer es concebido como creación de conocimiento, como una dinámica de autoconciencia -individual y colectiva- que permita reconocer las relaciones de opresión y facilitar la liberación.

\section{EL EUROCENTRISMO EN CUESTIÓN O LA COLONIALIDAD COMO EL LADO OSCURO DE LA MODERNIDAD}

Algunos de los asuntos principales de estas exploraciones anteriores han sido retomados más recientemente desde nuevas y fecundas perspectivas en la forma de un cuestionamiento global y sistemático a los supuestos coloniales y eurocéntricos de los saberes sociales de y sobre el continente. Se trata de un rico debate que expresa las condiciones en las cuales se da la producción político intelectual latinoamericana en la actualidad. Se trata en primer lugar de un debate que tiene como participantes activos a intelectuales latinoamericanos que trabajan desde América Latina, desde universidades norteamericanas, o frecuentemente combinando ambas localizaciones. Esto ha permitido mayores grados de diálogo e intercambio con intelectuales de otras regiones, en especial del Sur (Africa, la India, sureste asiático) que lo que ha sido característico del mundo académico del continente hasta el momento. De esta manera los debates sobre el postcolonialismo

\footnotetext{
${ }^{1}$ Darcy Ribeiro, Las Américas y la civilización. Proceso de formación y causas del desarrollo desigual de los pueblos americanos, Biblioteca Ayacucho, Caracas, 1988, p. 65. cirudo por Walter Mignolo. Local Histories/Global Designs. Coloniality, Subaltem Knowledges and Border Thinking, Princeton Lniversity Press, Princeton, 2000, p. 21.
} 
y los estudios subaltenos. las interpretaciones de la experiencia moderna desde el mundo excolonial y neo-colonizado, no son insumos bibliográficos. sino parte de confrontaciones e intercambios cotidianos en un ambiente intelectual culturalmente heterogéneo. En segundo lugar, este debate latinoamericano. lejos de ser la expresión de una nueva moda asociada a los estudios postcoloniales y estudios culturales que han tenido un inmenso auge en las universidades del Norte, está firmemente enraizado en las tradiciones intelectuales latinoamericanas a las cuales se ha hecho referencia, primer lugar donde se formularon muchos de los asuntos centrales de estos debates.: En tercer lugar, se parte del supuesto de que los procesos de descolonización del imaginario y del conocimiento requieren un doble movimiento, la crítica epistemológica a los saberes hegemónicos. y la producción de opciones alternativas prioritariamente desde lo subalterno. En consecuencia, este debate está de muchas formas articulado -directa o indirectamente-con movimientos populares que tienen entre sus objetivos la reivindicación de otras formas de conocimiento y modos de vida, como lo son. por ejemplo, los pueblos indígenas (Chiapas, Ecuador), el movimiento negro de la Costa Atlántica de Colombia, y el movimiento de los Sin Tierra en Brasil.

El propósito del resto de este texto es el de ilustrar la riqueza de este debate a partir de una presentación acotada de algunas de las contribuciones de cinco autores que han hecho aportes significativos a éste: Enrique Dussel, Aníbal Quijano, Walter Mignolo, Fernando Coronil y Arturo Escobar.

Son varios los ejes que articulan este debate en una perspectiva abierta, pero igualmente arecientemente coherente en sus líneas de cuestionamiento a los saberes hegemónicos, e incipientemente, en la propuesta de alternativas. Se abordan temas cruciales para la comprensión de las sociedades contemporáneas -en particular del mundo periférico- como la critica a las pretensiones universales de la historia local, parroquial europea; la polémica sobre el origen y rasgos esenciales de la modernidad; las relaciones entre modernidad y orden colonial; las condiciones históricas del surgimiento de los saberes modernos en los centros del proceso de la constitución del sistema-mundo moderno/colonial; la separación jerárquica entre los saberes abstractos, científicos, formales y los saberes locales y/o tradicionales; y el papel que han desempeñado los saberes modernos hegemónicos en la naturalización y legitimación del continuado proceso de colonización y neo-colonización tanto externa como interna de los pueblos del Sur.

Para Enrique Dussel un punto de partida necesario de esta discusión lo constituye la tarea de desmontar el mito eurocéntrico de la modernidad, afirmando para ello que es necesario reconocer que existen dos conceptos de modernidad.

El primero es eurocéntrico, provinciano, regional. La Modernidad es una emancipación, una 'salida' de la inmadurez por un esfuerzo de la razón como proceso crítico, que abre a la humanidad a un nuevo desarrollo del ser humano. Este proceso se cumpliría en Europa, esencialmente en el siglo XVIII. El tiempo y el espacio de este fenómeno lo describe Hegel, y lo comenta

\footnotetext{
De hecho existen diferencias significativas entre los intereses y las perspectivas del debate latinoamericano y orientaciones que Araviesan a las vertientes más difundidas del llamado debate postcolonial. Fernando Coronil, destaca dos hechos que -desde una ?erspectiva latinoamericana- llaman la atención en el campo académico metropolitano sobre la experiencia colonial: la concenración casi exclusiva en el colonialismo norte europeo en Africa y Asia y la ausencia del tema del imperialismo. Considera que :ilo "obliga a examinar las políticas del conocimiento en Occidente. a explorar las mancras como la teolía se difunde y a discernir - wo se establecen las nuevas modalidades colonizantes de influenciu en diferentes regiones y disciplinas académicas." "Natu-..teza del poscolonialismo: del eurocentrismo al globocentrismo", en Edgardo Lander (editor) La colonialidad del saber: : "rocentismo y ciencias sociales. Perspectivas latinoamericanas. LNESCO y Linversidad Central de Venezuela, Caracas (en ว
} 
Habermas en su conocida obra sobre el tema -y es unánimemente aceptado por toda la tradición europea actual.

...se sigue una secuencia espacio-temporal: casi siempre se acepta también el Renacimiento italiano. la Reforma y la Ilustración alemana y la Revolución francesa.... el Parlamento inglés. Es decir: Italia (siglo XV), Alemania (siglos XVI-XVIII), Francia (siglo XVIII), Inglaterra (siglo XVII). Denominamos a esta visión 'eurocéntrica' porque indica como punto de partida de la 'Modernidad' fenómenos intra-europeos, y el desarrollo posterior no necesita más que Europa para explicar el proceso. Esta es aproximadamente la visión provinciana y regional desde Max Weber -con su análisis sobre la 'racionalización' y el 'desencanto'- hasta Habermas. Para muchos un Galileo (condenado en 1616), Bacon (No'um Organum, 1620) o Descartes (El discurso del método, 1636) serian los iniciadores del proceso moderno en el siglo XVII. ${ }^{3}$

Proponemos una segunda visión de la 'Modernidad', en un sentido mundial, y consistiría en definir como determinación fundamental del mundo moderno el hecho de ser (sus Estados, ejércitos, economía, filosofía, etc.) 'centro' de la Historia Mundial. Es decir, nunca hubo empíricamente Historia Mundial hasta el 1492 (como fecha de iniciación del despliegue del 'Sistemamundo'). Anteriormente a esta fecha los imperios o sistemas culturales coexistían entre sí. Sólo con la expansión portuguesa desde el siglo XV, que llega al Extremo Oriente en el siglo XVI, y con el descubrimiento de América hispánica, todo el planeta se torna el 'lugar' de 'una sola' Historia Mundial...

Es esta una historia mundial moderna que se constituye, desde sus inicios en términos coloniales.

El ego cogito moderno fue antecedido en más de un siglo por el ego conquiro (Yo conquisto) práctico del hispano-lusitano que impuso su voluntad (la primera 'Voluntad-de-Poder' moderna) al indio americano. La conquista de México fue el primer ámbito del ego moderno.

Dussel caracteriza el "mito" de la modernidad en los siguientes términos:

a) La civilización moderna se autocomprende como más desarrollada, superior.

b) La superioridad obliga a desarrollar a los más primitivos, rudos, bárbaros, como exigencia moral.

c) El camino de dicho proceso educativo de desarrollo debe ser el seguido por Europa.

d) Como el bárbaro se opone al proceso civilizador, la praxis moderna debe ejercer en último caso la violencia si fuera necesario, para destruir los obstáculos de la tal modernización (la guerra justa colonial).

e) Esta dominación produce víctimas (de muy variadas maneras), violencia que es interpretada como un acto inevitable, y con el sentido cuasi-ritual de sacrificio; el héroe civilizador inviste a sus mismas víctimas del carácter de ser holocaustos de un sacrificio salvador (el indio colonizado, el esclavo africano, la mujer, la destrucción ecológica de la tierra, etcétera).

f) Para el moderno, el bárbaro tiene una 'culpa' (el oponerse al proceso civilizador) que permite a la 'Modernidad' presentarse no sólo como inocente sino como 'emancipadora' de esa 'culpa' de sus propias víctimas.

g) Por último, y por el carácter 'civilizatorio' de la 'Modernidad', se interpretan como inevitables los sufrimientos o sacrificios (los costos) de la 'modernización' de los otros pueblos 'atrasados' (inmaduros), de las otras razas esclavizables, del otro sexo por débil, etcétera. ${ }^{4}$

\footnotetext{
3 "Europa, modernidad y eurocentrismo", en Edgardo Lander (editor), op. cit. De la amplia producción de Dussel sobre estos temas. ver igualmente: 1492: El encubrimiento del Otro. Hacia el origen del mito de la Modernidad. Nueva Utopia, Madrid, 1992, y Etica de la Liberación en la Edad de la Globalización y la Exclusión, Editorial Trotta-UAM.I-UNAM. México, 1998.

4 Op. cit.
} 
Para superar la modernidad, de acuerdo a Dussel es indispensable primero "negar la negación" de este mito, reconocer la "otra cara" negada. oculta, pero no por ello menos esencial, la cara colonial de la modernidad, ya que lo que significó emancipación para unos fue el sometimiento para los "otros".

Al negar la inocencia de la 'Modernidad' y al afirmar la Alteridad de 'el Otro', negado antes como víctima culpable, permite 'des-cubrir' por primera vez la 'otra-cara' oculta y esencial a la 'Modernidad': el mundo periférico colonial, el indio sacrificado, el negro esclavizado, la mujer oprimida, el niño y la cultura popular alienadas, etcétera (las 'víctimas' de la 'Modernidad') como víctimas de un acto irracional (como contradicción del ideal racional de la misma Modernidad).

Anibal Quijano coincide con Dussel en el criterio de acuerdo al cual la modernidad se inicia con América, con la constitución del nuevo patrón de poder global, lo que Wallerstein ha llamado el sistema-mundo capitalista.

No se trata de cambios dentro del mundo conocido, que no alteran sino algunos de sus rasgos. Se trata del cambio del mundo como tal. Este es. sin duda. el elemento fundante de la nueva subjetividad: la percepción del cambio histórico. Es ese elemento lo que desencadena el proceso de constitución de una nueva perspectiva sobre el tiempo y sobre la historia. La percepción del cambio lleva a la idea del futuro, puesto que es el único territorio del tiempo donde pueden ocurrir los cambios... Con América se inicia. pues, un entero universo de nuevas relaciones materiales e intersubjetivas. ${ }^{5}$

...los cambios ocurren en todos los ámbitos de la existencia social de los pueblos y, por tanto de sus miembros individuales, lo mismo en la dimensión material que en la dimensión subjetiva de esas relaciones. Y puesto que se trata de procesos que se inician con la constitución de América, de un nuevo patrón de poder mundial y de la integración de los pueblos de todo el mundo en ese proceso, de un entero y complejo sistema-mundo, es también imprescindible admitir que se trata de todo un período histórico. En otros términos, a partir de América un nuevo espacio/ tiempo se constituye, material y subjetivamente: eso es lo que mienta el concepto de modernidad. $^{6}$

"Colonialidad del poder, eurocentrismo y América Latina", en Edgardo Lander (editor), op. cit. Los aportes más importantes de Quijano a este debate está recogjdos en los siguientes textos: "Modernidad y democracia: intereses y conflictos". Anuario Gariareguiano, vol XII, no. 12. Lima, 2000; "QQué tal raza!", en Familia y cambio social, CECOSAM, Lima. 1999; "Coloniality of power and its institutions", Documento aún no publicado del Simposio sobre Colonialidad del poder y sus ámbitos sociales, Binghamton University, Binghamton, Nueva York, abril 1999; "Estado nación, ciudadanía y democracia: cuestiones abiertas". en Helena González y Heidulf Schmidt (editores), Democracia para ma nueva sociedad, Nueva Sociedad, Caracas, 1998 , Colonialidad del poder, cultura y conocimiento en América Latina", en Amario Mariateguimo, vol. IX, no.9, Lima. 1997: 'Raza, etnia, nación: cuestiones abiertas", en Roland Forgues (editor), José Carlos Mariátegui y Europa. La otra cara del descubrimiento, Amauta, Lima, 1992: "Colonialidad y modernidad/racionalidid", en Perú Indigena, vol, 13. no. 29. 1992, Lima.

Colonialidad del poder eurocentrismo y América Latina". op. cit.

"En el proceso que llevó a ese resultado, los colonizadores ejercieron diversas operaciones que dan cuenta de las condiciones que lievaron a la configuración de un nuevo universo de relaciones intersubjetivas de dominación entre Europa y lo europeo y las demás regiones y poblaciones del mundo. a las cuales les estaban siendo atribuidas, en el mismo proceso, nuevas identidades reoculturales. En primer lugar, expropiaron a las poblaciones colonizadas -entre sus descubrimientos culturales- aquéllos que resultaban más aptos para el desarrollo del capitalismo y en beneficio del centro europeo. En segundo lugar, reprimieron tanto como pudicron, es decir en variables medidas según los casos. las formas de producción de conocimiento de los colonizados, sus patrones de producción de sentidos, su universo simbólico. sus patrones de expresión y de objetivación de lá subjetividad. La represión en este campo fue, conocidamente más violenta. profunda y duradera entre los indios de América ibérica. a los que condenaron a ser una subcultura campesina. iletrada. despojándolos de su herencia intelectual objetivada. Algo equivalente scurrió en Africa. Sin duda mucho menor fue la represión en el caso de Asia. en donde por lo tanto una parte importante de la historia y de la herencia intelectual, escrita. pudo ser preservada. Y fue eso. precisamente, lo que dio origen a la categoría de Oriente. En tercer lugar. forzaron -también en medidas variables en cada caso- a los colonizados a aprender parcialmente la cultura de los dominadores en todo lo que fuera útil para la reproducción de la dominación, sea en el campo de la actividad material tecnológica, como de la subjetiva. especialmente religiosa. Es este el caso de la religiosidad judeo-cristiana. Todo ese accidentado proceso implicó a largo plazo una coloniación dé las perspectivas cognititas. de los modos de producir u otorgar sentido a los resultados de la experiencia material o intersubjetiva del imaginario. del universo de relaciones intersubjetivas del mundo. de la cultura en suma". (Op. cit.) 
Este nuevo patrón mundial (colonial) del poder. es la condición en la cual se va constituyendo una nueva perspectiva (eurocéntrica) del conocimiento de la cual Quijano destaca los siguientes aspectos básicos:

En primer lugar, lugar se trata de una perspectiva de conocimiento sustentada sobre el dualismo radical cartesiano. que se convierte en una radical separación entre "razón/sujeto" y "cuerpo". a partir de lo cual el "cuerpo" fue naturalizado. fijado como "objeto" de conocimiento, por parte del "sujeto/razón". Esta separación (abstracción) del sujeto/razón en relación con el cuerpo está en la base de las pretensiones objetivistas y universalizantes de un saber (científico) ques reivindica su separación de los condicionamientos subjetivos (corporales), espaciales y temporales,

En segundo lugar, se produce en la perspectiva eurocéntrica del conocimiento una "articulación peculiar" entre el dualismo que establece contrastes radicales entre pre-capital y capital. entre lo no europeo y lo europeo, entre los primitivo y lo civilizado, entre los tradicional y lo moderno, por un lado, y la concepción evolucionista, lineal. unidireccional de la historia que avanza inexorablemente desde un mítico estado de naturaleza hasta la moderna sociedad europea.

En tercer lugar, a partir de las estructuras coloniales del poder, se naturalizan las diferencias culturales entre los grupos humanos mediante un sistemático régimen de codificación y clasificación de estas diferencias como diferencias raciales. "La idea de rasa [que] es. literalmente, un invento... [y que no] tiene nada que ver con la estructura biológica de la especie humana" se ha convertido en un extraordinariamente potente dispositivo de clasificación y jerarquización mediante el cual se ha logrado darle la apariencia de natural (y por lo tanto sin relación alguna con el orden social) a las profundas desigualdades y jerarquias existentes en las sociedades modernas. "En América, la idea de raza fue un modo de otorgar legitimidad a las relaciones de dominación impuestas por la conquista. La posterior constitución de Europa como nueva identidad después de América y la expansión del colonialismo europeo sobre el resto del mundo, Hevaron a la elaboración de la perspectiva eurocéntrica de conocimiento y con ella a la elaboración teórica de la idea de raza como naturalización de esas relaciones coloniales de dominación entre europeos y no-europeos. Históricamente, eso significó una nueva manera de legitimar las ya antiguas ideas y prácticas de relaciones de superioridad/inferioridad entre dominados y dominantes. Desde entonces ha demostrado ser el más eficaz y perdurable instrumento de dominación social universal. pues de él pasó a depender inclusive otro igualmente universal. pero más antiguo, $\mathrm{el}$ inter-sexual o de género: los pueblos conquistados y dominados fueron situados en una posición natural de inferioridad y, en consecuencia, también sus rasgos fenotípicos, así como sus descubrimientos mentales y culturales. De ese modo, raza se convirtió en el primer criterio fundamental para la distribución de la población mundial en los rangos, lugares y roles en la estructura de poder de la nueva sociedad. En otros términos, en el modo básico de clasificación social universal de la población mundial." Op. cit.

En cuarto lugar, a partir del dualismo y del evolucionismo arriba señalados, y a partir de la esencialización de las diferencias entre los pueblos como diferencias jerárquicas de capacidad humana, "los europeos imaginaron... ser no solamente creadores exclusivos de [la] modernidad, sino igualmente sus protagonistas." De esta manera se construye una Historia Universal en la cual todos los aportes significativos de las artes, las ciencias, la tecnología, la moral y los regímenes políticos son productos internos de la sociedad europeo, productores superiores a ser llevados al resto, inferior, de los pueblos del mundo.

En quinto lugar, como producto de la ubicación temporal de las diferencias entre los distintos pueblos, todo lo no-europeo es percibido como pasado. "Los pueblos colonizados eran razas inferiores y -por ello- anteriores a los europeos.

"La formación de relaciones sociales fundadas en dicha idea. produjo en América identidades sociales históricamente nuevas indius, negros y mestizos y redefinió otras. Así términos como español y portugués, más tarde curopeo. que hasta entonces indicaban solamente procedencia geográfica o país de origen. desde entonces cobraron también. en referencia a las nuevas identidades, una connotación racial. Y en la medida en que las relaciones sociales que estaban configurándose eran relaciones do dominación, tales identidades fueron asociadas a las jerarquias, lugares y roles sociales correspondientes. como constitutivas de ellas y, en consecuencia. al patrón de dominación colonial que se imponia. En otros términos. raza e identidad racial fueron establecidas como instrumentos de clasificación social básica de la población." Op. cit. 
La importancia actual de estos asuntos reside en que si bien esta estructuri de poder (y el régimen de saberes caracterizado como eurocéntrico) tiene un origen colonial, "ha aprobado ser más duradero y estable que el colonialismo", perdurando como un elemento del patrón de poder hoy mundialmente hegemónico.

Fernando Coronil ofrece una poderosa perspectiva para la crítica a las concepciones eurocéntricas de la modernidad y del desarrollo del capitalismo (Occidentalisno) aportando de esa forma instrumentos para el proyecto de la paroquiali-ación de la modemidad occidental." Se trata de la exploración de sus ausencia 1 olvidos. lo que teoría social moderna deja afuera de su mirada en la caracterización de esta sociedadi el espacio y la naturale -a. Afirma que "los historiadores y los científicos sociales usualmente presentan al espacio o a la geografía como un escenario inerte en el cual tienen lugar los eventos históricos. y a la naturaleza como el material pasivo con el cual los humanos hacen su mundo." La exclusión del espacio y de la naturaleza está inseparablemente imbricado de las concepciones de la modernidad y del capitalismo como productos intemos de la historia europea que posteriormente fueron propagados o llevados al resto del planeta. a la comprensión de la modernidad como "creación de un Occidente autopropulsado"to y Europa "como portadora universal de la razón y del progreso histórico". El proyecto de la parroquialización de la modernidad exige la comprensión de la modernidad y del capitalismo, desde sus inicios. como un proceso global. implica, en el cual lejos de existir un único locus (Europa occidental), hay un proceso global, crecientemente planetario en el cual la periferias colonizadas lejos de ser "la encarnación del atraso bárbaro" son la expresión subalterna de la modernidad. ${ }^{\text {" }}$

Una apreciación del papel de la naturaleza en la creación de riqueza ofrece una visión diferente del capitalismo. La inclusión de la naturaleza (y de los agentes asociados con ésta) debería reemplazar a la relación capital/trabajo de la centralidad osificada que ha ocupado en la teoria marxista. Junto con la tierra, la relación capital/trabajo puede ser vista dentro de un proceso más amplio de mercantilización, cuyas formas específicas y cfectos deben ser demostrados concretamente en cada instancia. A la luz de esta visión más comprensiva del capitalismo, sería difícil reducir su desarrollo a una dialéctica capital/trabajo que se origina en los centros avanzados y se expande a la periferia atrasada. Por el contrario, la división internacional del trabajo podría ser reconocida más adecuadamente como simultáneamente una división internacional de naciones y de naturaleza ( $y$ de otras unidades geopolíticas, tales como el primer y el tercer mundo. que reflejan las cambiantes condiciones internacionales). Al incluir a los agentes que en todo el mundo están implicados en la creación del capitalismo, esta perspectiva hace posible vislumbrar una concepción global, no eurocéntrica de su desarrollo. ${ }^{12}$

Incorporar la naturaleza y el espacio a la teoría, es incorporarlas a la comprensión del desarrollo capitalista, es comprender el rol de la naturaleza en la creación de riqueza y ubicar el desarrollo capitalista y sus agentes no sólo al interior de Europa sino igualmente incorporando desde sus inicios -por la vía colonial- a los territorios, poblaciones y recursos del resto del planeta.

La inclusión de agentes mundiales involucrados en la aparición del capitalismo ayuda a desarrollar un recuento descentrado de la historia. Desde tiempos coloniales, la "periferia" ha sido una fuente principal tanto de riquezas naturales como de trabajo barato.

Integrar la "tierra" a la relación capital/trabajo ayuda a comprender los procesos que le dieron forma a la constitución mutua de Europa y sus colonias. En vez de una narrativa de la historia construida en términos de una oposición entre una Europa moderna que ha triunfado por su

Además del texto ya citado, ver: The Magical State. Nature. Money and Modernity in Veneztela. Chicago University Press, Chicago, 1997, y 'Beyond Occidentalism: towards Tonimperial Geohistorical categories' Cultural Anthropology; Vol. 11, No. 1, 1996.

The Magical State. Nature, Money and Modemity in Veneruela, op. cit. p. 23

Op. cit., p. 7.

Op. cit.. p. 74

Op. cit. p. 61 
propio esfuerzo, y una periferia atrasada, este cambio de perspectiva nos permite apreciar más cabalmente el rol de la naturaleza (neo) colonial y el trabajo en la formación transcultural de las modernidades metropolitanas y subalternas (Ortiz 1995: Coronil 1995:1997).

De acuerdo con Coronil, hoy es necesario ir más allá de la crítica al eurocentrismo que ha buscado provincializar la modernidad, con la crítica al globocentrismo que "debería buscar diferenciar el mundo y mostrar la altamente desigual distribución de poder y la inmensa diversidad cultural".

El globocentrismo, como modalidad del Occidentalismo, también se refiere a prácticas de representación implicadas en el sometimiento de las poblaciones no occidentales, pero en este caso su sometimiento (igual que el sometimiento de sectores subordinados dentro de Occidente) aparece como un efecto del mercado, en vez de como consecuencia de un proyecto político (occidental) deliberado. En la medida que el 'Occidente' se disuelve en el mercado, se funde y solidifica a la vez.

La magia del imperialismo hoy reside en conjurar su propia desaparición haciendo que el mercado aparezca como la personificación de la racionalidad humana y de la felicidad. Los discursos dominantes de globalización ofrecen la ilusión de un mundo homogéneo que avanza constantemente hacia el progreso. Pero la globalización está intensificando las divisiones en la humanidad y está acelerando la destrucción de la naturaleza. Es tarea de los estudiosos críticos postcoloniales desenmascarar este truco mágico.

De acuerdo a Walter Mignolo ${ }^{3}$, en la constitución del mundo colonial a partir de la conquista de los territorios americanos, se instaura un lugar privilegiado de creación de sentido y de conocimiento articulado a esa estructura de poder. Es lugar es un privilegiado locus de cuntuciación, entendido en términos tanto físicos como teóricos.

La construcción de una perspectiva occidental fue la construcción de un lugar de enunciación maestro en el cual la concepción misma de la historia universal y de su escritura estaban inscritos en la expansión colonial y legitimaron su aparato imperial y de Estado-nación. ${ }^{14}$

Lat dialéctica de los europeos como purticipantes en el proceso de colonización y los europeos como observadores $d e l$ proceso fue constante y persistente. Como participantes. los españoles y europeos en general vivían y actuaban de acuerdo a sus metas. deseos y necesidades a partir de un marco de referencia conceptual (o conjunto de marcos de referencia). Como observadores, los literatos españoles y europeos se convirtieron en los jueces capaces de comparar y evaluar marcos conceptuales inconmensurables. Uno de los puntos cruciales en la construcción de la otredad fue, precisamente. ente movimiento oculto entre el describirse a sí mismo como perteneciendo a un marco de referencia dado, $y$ la descripción como perteneciente al marco de referencia correcto. (...) Al desempeñar ambos papeles al mismo tiempo. los intelectuales europeos fueron capaces de implementar (desde Europa o desde el Nuevo Mundo) sus descripciones como observadores y enlazarlas con el ejercicio del poder imperial.

El proceso de expansión colonial que comenzó en el siglo XVI corre paralelo a la creciente consolidación del sujeto del conocimiento y la comprensión en una geografía dada, construida sobre las ruinas de dos lenguas occidentales asociadas al conocimiento y a la sabiduría. (el griego y el latín) y situadas en una idea creciente de tiempos de referencia progresi vos o evolucionarios. Tales desarrollos negaban la posibilidad de que otros lugares de enunciación e historias co-

\footnotetext{
1: Las contribuciones principales de Walter Mignolo están recogidas en texto ya citado y en: The Daker Side of the Renaissance The University of Michigan Press. Ann Arbor. 1905

It The Darker Side of the Renaissance. p. 329

${ }^{15}$ Op. cit., p. 328.
} 
evolutivas fuesen posibles. Desde el período moderno temprano hasta el siglo XVIII la consolidación de una forma de conocimiento como espejo de la naturaleza se fue estableciendo. Mi supuesto en este libro es que tal consolidación de formas de conocer implica la complicidad entre el lugar de enunciación regional de un participante de la cultura europea occidental y el lugar de enunciación universal de la ciencia y la filosofía por parte de un sujeto que está colocado fuera del tiempo y el espacio. Es esto precisamente la universalización de conceptos regionales de ciencia. filosofía $y$ conocimiento. ${ }^{16}$

Walter Mignolo parte de la "profunda convicción"de que una de las ricas avenidas de la ieorización postcolonial es precisamente, el abrir posibilidades para diversos y legítimos lugares de enunciación teóricos, y que al hacerlo, permite relocalizar al sujeto monológico e universal del conocimiento, inscrito en el período moderno/colonial. ${ }^{17}$ A partir del supuesto de que las alternativas a la epistemología moderna difícilmente pueden provenir sólo de la epistemología occidental) modema misma ${ }^{18}$, en la búsqueda de esta apertura a otros lugares legítimos de enunciación, propone un los conceptos relacionados de diferencia colonial y pensamiento desde al borde (border thinking).

Por 'diferencia colonial' quiero decir... la clasificación del planeta en el imaginario modernol colonial, mediante la colonialidad del poder, una energía y una maquinaria para transformar diferencias en valores. Si el racismo es la matriz que permea cada dominio del imaginario del sistema-mundo moderno/colonial, occidentalismo es la metáfora omnipresente en torno a la cual las diferencias coloniales han sido articuladas y re-articuladas a través de las manos cambiantes de la historia del capitalismo... y las cambiantes ideologías motivadas por los conflictos coloniales. ${ }^{19}$

Es también el lugar en el cual está teniendo ligar la restitución del conocimiento subalterno y en el cual emerge el pensamiento de borde. La diferencia colonial es el espacio en el cual historias locales inventando e implementando diseños globales, se encuentran con historias locales, el espacio en el que los diseños globales tienen que ser adaptados, adoptados, rechazados, integrados o ignorados. ${ }^{20}$

La diferencia colonial crea las condiciones de situaciones dialógicas en que las enunciaciones fracturadas son llevadas a cabo (enacted) desde una perspectiva subalterna como una respuesta al discurso y perspectiva hegemónica. ${ }^{21}$

Destaca Mignolo la existencia de un potencial epistémico de pensamiento de borde (desde una perspectiva subalterna)que emerge de las grietas entre civilización y cultura, entre globalización y 'mundialización', entre los diseños globales y las culturas locales. Este conocimiento desde los bordes no pretende rescatar las autenticidades de otras culturas, ni la "representación" de lo invisible. A diferencia de la epistemología hegemónica, con su énfasis en la denotación y la serdad, para el conocimiento de borde el énfasis está en la performatividad y en la transformación. 22

El conocimiento de borde es un conocimiento desde dos (o más) historias locales entretejidas por la colonialidad del poder. Es a la vez una doble crítica -desde el borde- de los conocimientos I las epistemologías correspondientes a cada una de esas historias locales. Es un conocimiento en curre cuando el imaginario del sistema-mundo moderno se resquebraja. La postura desde el borde, la experiencia de las identidades fragmentadas, la pertenencia simultánea a más de un

\footnotetext{
Op. cit., p. 330 .

The Darker Side of the Renaissance, (p. ix).

- Local Histories/Global Designs, p. 9.

Op. cit., p. 13.

Op. cit. . p. ix

Op. cit. p. $x$.

Op. cit. p. 24
} 
universo cognitivo (diferencia colonial, migración exilio) hace posible la crítica simultánea de las cosmovisiones, de dichos epistemes. Se hace posible desde la ubicación simultánea en estas dos historias locals, la construcción de enunciados universales. "... 'un otro pensamiento' es una historia universal del sistema mundo moderno/colonial que implica la complementariedad de la modernidad y la colonialidad, el moderno colonialismo (desde 1500 y sus conflictos internos) y las modemidades coloniales, en sus diversos ritmos, temporalidades, en que naciones y religiones entran en conflicto en diferentes períodos y en diferentes órdenes mundiales." 23

De los trabajos de Arturo Escobar interesa destacar sólo algunas líneas de indagación que con particularmente pertinentes para los asuntos que aquí se discuten ${ }^{2+}$. En primer lugar, está su contribución a la construcción de un marco de referencia para la crítica cultural de la economía, como estructura fundacional de la modernidad orientado a historizar, a desnaturalizar lo que en el pensamiento hegemónico aparecen como "la realidad" universal.

Si con al modernidad podemos hablar de la progresiva conquista semiótica de la vida social y cultural, hoy esta conquista se ha extendido al corazón mismo de la naturaleza y la vida. Una vez que la modernidad se ha consolidado y la economía se convierte en una realidad aparentemente ineluctable -para la mayoría un verdadero descriptor de la realidad- el capital debe abordar la cuestión de la domesticación de todas las relaciones sociales y simbólicas restantes en términos del código de la producción. Ya no son solamente el capital y el trabajo per se los que están en juego, sino la reproducción del código. La realidad social se convierte, para tomar prestada la expresión de Baudrillard, en 'el espejo de la producción'. 25

Plantea Escobar la necesidad de una antropología de la modernidad, que permita desnaturalizar al orden de la sociedad capitalista como universal y única posible.

La economía occidental es generalmente pensada como un sistema de producción. Desde la perspectiva de la antropología de la modernidad, sin embargo, la economía occidental debe ser vista como una institución compuesta por sistemas de producción, poder y significación. Los tres sistemas se unieron al final del siglo dieciocho y están inseparablemente ligados al desarrollo del capitalismo y la modernidad. Deben ser vistos como formas culturales a través de las cuales los seres humanos son transformados en sujetos productivos. La economía no es sólo, ni siquiera principalmente, una entidad material. Es ante todo, una producción cultural, una forma de producir sujetos humanos y órdenes sociales de un determinado tipo. ${ }^{26}$

Insistiendo en las implicaciones de las ausencias a las cuales habíamos hecho referencia arriba en la discusión de los trabajos de Fermando Coronil, Arturo Escobar argumenta que son múltiples las implicaciones de la exclusión del espacio en la teoría social hegemónica.

Un aspecto final de la persistente marginalización del lugar en la teoría occidental es el de las consecuencias que ha tenido en el pensar de las realidades sometidas históricamente al colonialismo occidental. El dominio del espacio sobre el lugar ha operado como un dispositivo epistemológico profundo del eurocentrismo en la construcción de la teoría social. Al restarle énfasis a la construcción cultural del lugar al servicio del proceso abstracto y aparentemente universal de la formación del capital y del Estado, casi toda la teoría social convencional ha hecho invisibles formas subalternas de pensar y modalidades locales y regionales de configurar

\footnotetext{
${ }^{2}$ Op. cit., p. 74

${ }^{24}$ Ver, especialmente: Encountering Development. The Making and Ummaking of the Third World. Princeton University Press, Princeton, 1995. (Edición en español: La invención del Tercer Mundo. Construcción y deconstrucción del desarrollo, Editorial Norma, Bogotá, 1998), y "El lugar de la naturaleza y la naturaleza del lugar: ¿globalización o postdesarrollo?", en Eduardo Lander (editor), op. cit.

${ }^{2 s}$ Encountering Development. The Making and Unmaking of he Third World, op. cit., p. 203.

${ }^{26}$ Op. cit., p. 59.
} 
el mundo. Esta negación del lugar tiene múltiples consecuencias para la teoría -desde las teorías del imperialismo hasta aquéllas de la resistencia. el desarrollo. etc.- que pudiesen ser exploradas mejor en el ámbito ecológico. En este ámbito. la desaparición del lugar está claramente vinculada al la invisibilidad de los modelos culturalmente específicos de la naturaleza y de la construcción de los ecosistemas. Solamente en los últimos años es cuando nos hemos dado cuenta de este hecho. ${ }^{27}$

Para Escobar la superación del eurocentrismo que caracteriza al conocimiento hegemónico pasa por poner en cuestión las múltiples separaciones sobre el cual este esté sustentado. Destaca para ello la diferencia entre la forma en la cual se asume la relación no sólo la dicotomía entre naturaleza y sociedad, sino igualmente la separación entre lo humano y lo supernatural prevaleciente el conocimiento moderno occidental dominante, $y$ las formas en las cuales se piensa esta relación en las múltiples y diversas expresiones del "conocimiento local".

Quizás la noción más arraigada hoy en día es que los modelos locales de la naturaleza no dependen de la dicotomía naturaleza/sociedad. Además, y a diferencia de las construcciones modernas con su estricta separación entre el mundo biofísico, el humano y el supernatural, se entiende comúnmente que los modelos locales, en muchos contextos no occidentales, son concebidos como sustentados sobre vínculos de continuidad entre las tres esferas. ${ }^{20}$

Las teorías sociales sustentadas sobre la prioridad del espacio sobre el lugar, la cultura global sobre las culturas locales, el conocimiento abstracto universal sobre los conocimientos jocales contribuyen a la naturalización, y por esa vía refuerzan al orden capitalista como el único posible.

El capitalismo ha sido investido de tal predominancia y hegemonía, que se ha hecho imposible pensar la realidad social de otra manera, mucho menos imaginar la supresión del capitalismo; todas las otras realidades (economías subsistentes, economías biodiversificadas, formas de resistencia del Tercer Mundo, cooperativas e iniciativas locales menores) son vistas como opuestas, subordinadas o complementarias al capitalismo, nunca como fuentes de una diferencia económica significativa. ${ }^{29}$

Un aspecto vital de las nuevas perspectivas de la crítica al eurocentrismo y al occidentalismo ve refiere a la dimensión que va más allá de la crítica de la Historia Universal, de los conocimientos de las humanidades y de las ciencias sociales, para abordar el ataque de la inexpugnable fortaleza de la objetividad y de la universalidad, el terreno de la supremacía incuestionada de Occidente: has ciencias naturales y las tecnologías modernas. Aunque menos elaborado como crítica y como propuesta, e igualmente -en la mayor parte de los autores trabajados en este texto-con conexiones menos directas con experiencias sociales alternas y de borde en los cuales se están llevando a zabo y recuperando prácticas de conocimiento y de tecnología alternativas son estos asuntos que son crecientemente reconocidos como importantes para este debate. Como con relación a otros usuntos, es fuerte todavía el peso de la tradición de las separaciones académicas y fraccionamiento Jisciplinarios heredados de la cosmovisión liberal. Los temas ecológicos y ambientales, los : aferidos a estilos de desarrollo, las disciplinas de las ciencias naturales y lo referido a las opciones :acnológicas caminan en términos tanto teóricos como prácticos por caminos diferentes con : lativamente pocos cruces y poca fecundación mutua con la producción de las ciencias sociales las humanidades. ${ }^{30}$ Sin una ruptura con estos moldes disciplinarios, avanzando por separado en

El lugar de la naturaleza y la naturaleza del lugar: ¿globalización o postdesarrollo?", en Eduardo Lander (editor), op. cit. Op. cit.

Op. cit.

Como expresión de una rica producción etnográfica y práctica sobre "otros" saberes y tecnologías, ver, por ejemplo los trabajos -uhlicados en la revista Hombre y Ambiente. El punto de vista indigena, publicada por la editorial Abya-Yala en Quito. 
cada una de las "dos culturales" será más difícil superar la naturalización de las relaciones sociales existentes y mucho mayores los obstáculos que se encontraran en el camino de la descolonización.

No se trata sin embargo de carencias no reconocidas, aquello a lo que se refiere Mignolo caracteriza como "el esfuerzo por desacoplarse de la tiranía de la razón occidental, sus ciencias y sus tecnologías..." 31 Para Escobar esto exige superar la separación que se establece en los saberes occidentales entre cultura y naturaleza y entre el conocer y el hacer. Destacando los trabajos de Humberto Maturana y Francisco Varela sobre las bases biológicas del conocimiento, afirma:

Al rechazar la separación del conocer y el hacer, y estas de la existencia, estos biólogos nos ofrecen un lenguaje con el que se puede cuestionar radicalmente las relaciones binarias y las asimetrías de la naturaleza y la cultura, y la teoría y la práctica; también corroboran las percepciones agudas de aquellos que documentan etnográficamente la continuidad entre la naturaleza y la cultura, y los aspectos corporeizados del conocimiento, como en las ideas de desarrollo de habilidades y performatividad. ${ }^{32}$

Argumenta que es abundante la investigación de acuerdo a la cual muchas comunidades rurales del Tercer Mundo "construyen" las relaciones entre cultura y naturaleza en forma diferente a la occidental, utilizan los ambientes naturales en formas diversas y particulares. Pareciera existir igualmente, afirma Escobar, una cierta convergencia en que el conocimiento local funciona como una actividad práctica, situada, como conocimiento corporeizados, más que como sistemas formales libres de contexto. Esto tiene implicaciones vitales para los debates en torno a los límites de la civilización industrial y para la búsqueda de opciones a este modelo de vida que está amenaza las condiciones que hacen posible la vida en el planeta Tierra.

[Enrique] Leff, en particular, defiende la incorporación de criterios culturales y tecnológicos a un paradigma alternativo de producción que va mucho más allá de la racionalidad económica dominante. Leff insiste que si es verdad que la sustentabilidad debe basarse en las propiedades estructurales y funcionales de los distintos ecosistemas, cualquier paradigma de producción alternativa conducente a ello debe incorporar las actuales condiciones culturales y tecnológicamente específicas bajo las cuales actores locales se apropian de la naturaleza. "El desarrollo sustentable encuentra sus raices en condiciones de diversidad cultural y ecológica. Estos procesos singulares y no reducibles dependen de las estructuras funcionales de ecosistemas que sustentan la producción de recursos bióticos y servicios ambientales; de la eficiencia energética de los procesos tecnológicos; de los procesos simbólicos y formaciones ideológicas que subyacen en la valorización cultural de los recursos naturales; y de los procesos políticos que determinan la apropiación de la naturaleza". 33 Dicho de otra manera, la construcción de paradigmas alternativos de producción, órdenes políticos, y sustentabilidad son aspectos de un mismo proceso, y este proceso es impulsado en parte por la política cultural de los movimientos sociales y de las comunidades en la defensa de sus modos de naturaleza/cultura. ${ }^{3+}$

${ }^{31}$ Encountering Development. The Making and Unmaking of the Third World, op. cit. p. 74.

32 "El lugar de la naturaleza y la naturaleza del lugar: ¿globalización o postdesarrollo?", op cit.

${ }^{33}$ Leff, Enrique: '¿De quién es la naturaleza'? Sobre la reapropriación social de los recursos naturales'. Gaceta Ecológica $37,1995$. p. 61

34 "El lugar de la naturaleza y la naturaleza del lug̣ar: ¿globalizacion o postdesartollo?", en Eduardo Lander (cditor), op. cit. 


\section{CONCLUSIONES}

Si algo queda claro en este conjunto de planteamientos, es que las separaciones de los ámbitos de la realidad y de los saberes disciplinarios heredades del liberalismo, son una dimensión principal de las estructuras cognitivas de las cuales es necesario liberarse. Estos ámbitos disciplinarios fraccionados no pueden operar sino como dispositivos de naturalización de "la realidad" del sistema-mundo moderno colonial-imperial. De los debates actuales a los cuales se hace referencia breve en este texto es posible concluir igualmente que la descolonización con relación al imaginario y los saberes eurocéntrico hegemónicos, no es sólo una condición sin la cual no será posible un cambio en las condiciones de subordinación y exclusión en las cuales vive la mayor parte de la población del planeta, sino que constituye igualmente una condición sin la cual difícilmente pueda caminarse hacia otro(s) modelo(s) civilizatorio(s) que garanticen la continuidad de la vida en este planeta que "todos compartimos provisionalmente". 35 De ahí la importancia de este debate, a pesar de que estos asuntos todavía se encuentren al margen de las tendencias predominantes de la producción académica y política del continente.

Fernando Coronil. "Naturaleza del postcolonialismo: del eurocentrismo al globocentrismo". 